\title{
CRYSTALLIZATION IN COUETTE-TAYLOR CRYSTALLIZER: EFFECT OF TAYLOR VORTICES FLOW ON THE NUCLEATION AND RECONSTRUCTION OF L-GLUTAMIC ACID IN COOLING CRYSTALLIZATION
}

\author{
Khuu Chau Quang', Dang Truong Giang ${ }^{1}$, Nguyen Dinh Tuyet ${ }^{1}$, Le Thi Hong Nhan ${ }^{2}$, \\ Phan Thanh Thao", Trinh Thi Thanh Huyen ", , Nguyen Anh Tuan", \\ ${ }^{1}$ Institute of Chemical Technology (VAST), 1 Mac Dinh Chi Street, District 1, HCMC \\ ${ }^{2}$ HoChiMinh City University of Technology, 268 Ly Thuong Kiet Street, District 10, HCMC \\ "Email: thanhhuyenkhtn@yahoo.com,nhtnat@yahoo.com
}

Received: 26 November 2015; Accepted for publication: 26 January 2016

\begin{abstract}
The effect of Taylor vortices flow of Couette-Taylor (CT) crystallizer on the nucleation and reconstruction of L-glutamic acid was firstly investigated in cooling crystallization. Generally, L-glutamic acid has two kinds of polymorphic crystal including metastable $\alpha$-form and stable $\beta$ form, where the $\alpha$-form is initially crystallized and then slowly transformed into the $\beta$-form, which is called the phase transformation. The present study explored that there was a significant difference between CT and the conventional ST crystallizer as regards the nucleation and reconstruction of L-glutamic acid. Here, the phase transformation determined by the nucleation and reconstruction was $40 \mathrm{~h}$ in ST crystallizer, yet it was only $20 \mathrm{~h}$ as using the CT crystallizer, implying that the nucleation and reconstruction of L-glutamic acid was facilitated 2.0 times as using the Taylor vortices flow. The advantage of Taylor vortices flow in CT crystallizer over the random fluid motion in ST crystallizer with regards the nucleation and reconstruction was explained in term of the high energy dissipation of Taylor vortices flow.
\end{abstract}

Keywords: crystallization, nucleation, crystal growth, Couette-Taylor crystallizer, Taylor vortices flow.

\section{INTRODUCTION}

Crystallization is significant separation, purification and particle technology that has a wide application for a number of crystal products in various industries including chemicals, foods and pharmaceuticals, etc. Therefore, the crystallization processes are certainly required in order to obtain crystal products with the high qualities including purity, shape, polymorphism, size and size distribution, etc [1]. Despite its long history, crystallization is still not well understood as it involves many complex mechanisms of each material such as nucleation, crystal growth, fine dissolution, agglomeration, etc. Polymorphism is an interesting phenomenon in crystallization, 
where the same material can exist in more than one crystal structure due to the different arrangement of molecules in crystal lattice. Since the different crystal structure has the different physical and chemical properties including bioactivity, dissolution rate, solubility, hardness, etc, the polymorphism phenomenon is crucial in any crystallization process [2].

Amino acids are valuable materials with a wide application in numerous products including food, fine chemical, agricultural, cosmetic, and pharmaceutical products, etc. Thus, controlling of properties of amino acid crystals is significantly important [3 - 6]. In the present study, the amino acid L-glutamic was chosen as a model crystal product to demonstrate how to design an efficient crystallization process to control the polymorphism. Generally, L-glutamic acid has two kinds of polymorphic crystal including metastable $\alpha$-form and stable $\beta$-form, where controlling of each form is very complicated depending on many crystallization conditions [1 - 11]. Since the $\beta$-form was the desired stable crystal structure, many crystallization studies have focused on controlling of $\beta$-form crystal product. For example, Florence et al [3] reported that the Oscillatory baffled crystallizer (OBC) could be used to produce the $\beta$-form crystal product as the continuous crystallization system. Zhang et al [4] indicated that the 3D vision imaging technique was more valuable to capture the real shape and size of $\beta$-form crystal product compared to the conventional $2 \mathrm{D}$ vision imagining technique during crystallization, allowing provide a deep background mechanism of nucleation and crystal growth $\beta$-form. Meanwhile, Ochsenbein et al [5] demonstrated that the population balance model method was useful to estimate the growth rate of various surfaces and crystal size of $\beta$-form, so the fundamental growth mechanism of $\beta$ form is more understood.

Taylor vortices flow is a unique fluid hydrodynamic condition composed of a series turbulent circular fluid motion in the gap cylinders of CT crystallizer [6 - 10]. Thus, the Taylor vortices flow has already provided so many interesting crystallization phenomena that are never found in the other conventional crystallization processes [6 - 10]. For example, Tuan et al [6 - 8] reported that the phase transformation of GMP was significantly promoted at least 5.0 times as using the CT crystallizer compared to that of the conventional ST crystallizer. Park et al [9] also indicated that the nucleation rate of stable phase Sulfamerazine was more facilitated than that of the ST crystallizer, allowing accelerate the phase transformation of metastable phase into stable phase. Meanwhile, Mayra et al [10] indicated that the Taylor vortices flow was really effective to produce the spherical agglomerated battery material.

In Vietnam, although there are some researches relating partly with the crystallization studies, these crystallization studies have not been published on any ISI journals due to the lack of background and new ideas. In case of Couette-Taylor crystallizer, it is definitely noted that our patent Couette-Taylor crystallizer is firstly implemented in Vietnam. For L-glutamic, even though Glutamic acid is commercial by the Vedan company, this crystal product has extremely low quality with regard to the pharmaceutical product because the purity of this product is only $93-97 \%$ less than the standard purity as $98 \%$. Plus, the $\alpha$-form or $\beta$-form crystal and L- or Dglutamic acid are not clearly mentioned, implying that the crystallization process of this company is not well controlled. Indeed, this product is purposefully used as the raw material for the Glutamate sodium synthesis, and of course, it is not required having a high quality crystal product. In the foreign countries, although the L-glutamic acid crystallization has already carried out, it still has a lot of problems. For example, the phase transformation of $\alpha$-form into $\beta$-form consumed a long time as using the ST crystallizer, while the encrustation or blockage is often the problems as using the OBC crystallizer [4], etc. Therefore, a new crystallization process is really essential to develop. In the present study, the Couette-Taylor crystallizer with a unique fluid 
hydrodynamic Taylor vortices flow was firstly implemented to promote the nucleation and reconstruction of L-glutamic acid.

\section{EXPERIMENTAL}

The Couette-Taylor crystallizer (CT) and the conventional ST crystallizer (ST) were designed according to Tuan et al [6 - 8]. The temperature of crystallizer was controlled via the circulating coolant from the chiller. The L-glutamic acid material (98\% purity) was purchased from Sigma Aldrich. The feed solution was prepared by dissolving the material into the distilled water at $50{ }^{\circ} \mathrm{C}$, where the feed concentration was $18.5(\mathrm{~g} / \mathrm{L})$. The CT and ST crystallizer were initially filled with the feed solution at $50{ }^{\circ} \mathrm{C}$, and then operated as the batch mode crystallization with $4.0{ }^{\circ} \mathrm{C} / \mathrm{min}$ cooling rate.

The samples were periodically taken from the crystallizers and quickly filtered by using a vacuum pump. The crystal samples were then dried in a desiccator and analyzed to define the shape, structure and crystal fraction of $\beta$-form. Here, the shape and structure of crystal product was monitored and confirmed by Video microscope and XRD patterns (M18XHF-SRA, Japan), respectively. Meanwhile, the temperature was detected by the temperature indicator (Korea).

\section{RESULTS AND DISCUSSION}

\subsection{Polymorphism of L-glutamic acid}

The shape of $\alpha$-form and $\beta$-form crystals was clearly different as prism and needle, respectively, as shown in Fig. 1. Moreover, the crystal structure of each form was obviously distinguished via the XRD pattern at $10^{0}, 15^{0}, 16^{0}, 18^{0}, 21^{0}, 23^{0}, 26.5^{0}, 27.5^{0}$ degrees (Fig. 1). Plus, the mass fraction of $\alpha$-form and $\beta$-form was estimated via the FT-IR spectroscopy [11]. As shown in Fig. 2, the solubility of $\alpha$-form was always higher than that of $\beta$-form in a whole range of temperature, implying that the $\alpha$-form and $\beta$-form were the metastable and stable phase, respectively, and the $\alpha$-form transformed into the $\beta$-form during crystallization. Here, the driving force of phase transformation was the solubility gap between $\alpha$-form and $\beta$-form.

\subsection{Nucleation and reconstruction of L-glutamic acid in ST and CT crystallizer}

The typical phase transformation of L-glutamic acid was carried out in both ST and CT crystallizer, as shown in Figs. 3 - 4. In ST crystallizer, the solute concentration decreased from the feed concentration to the $\alpha$-form solubility after $5 \mathrm{~h}$, and then invaried until $20 \mathrm{~h}$. After that, the solute concentration continuously decreased to $\beta$-form solubility after $40 \mathrm{~h}$. This result revealed that the phase transformation completed after $40 \mathrm{~h}$, as depicted in Fig. 3. This concentration profile was consistent with the mass fraction of $\beta$-form profile, where the $\beta$-form was detected at $20 \mathrm{~h}$, meaning that the $\alpha$-form transformed into the $\beta$-form and the solid product was the mixture of $\alpha$-form and $\beta$-form. The mass fraction of $\beta$-form increased to the $100 \mathrm{wt} \%$ when the crystallization time was over $40 \mathrm{~h}$ (Fig. 3). The phase transformation of L-glutamic acid was also visually confirmed via the shape of solid product, as displayed in Fig. 3. Here, the only prism shape of $\alpha$-form was observed until $5 \mathrm{~h}$, while the mixture shape including prism and needle was captured as the crystallization time varied from $5 \mathrm{~h}$ to $40 \mathrm{~h}$, implying that the solid product was the mixture of $\alpha$-form and $\beta$-form until $40 \mathrm{~h}$. The entire needle shape of solid 
product corresponding to the pure $\beta$-form was only observed after $40 \mathrm{~h}$, indicating that the phase transformation completed after $40 \mathrm{~h}$ as using the ST crystallizer.
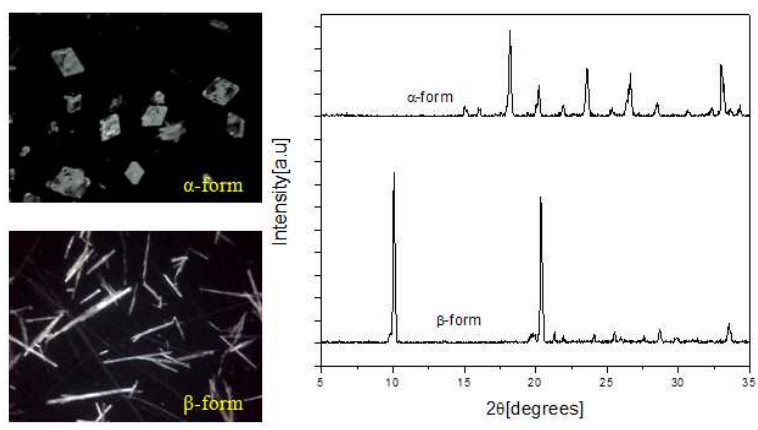

Figure1. Shape and structure of $\alpha$-form and $\beta$-form.

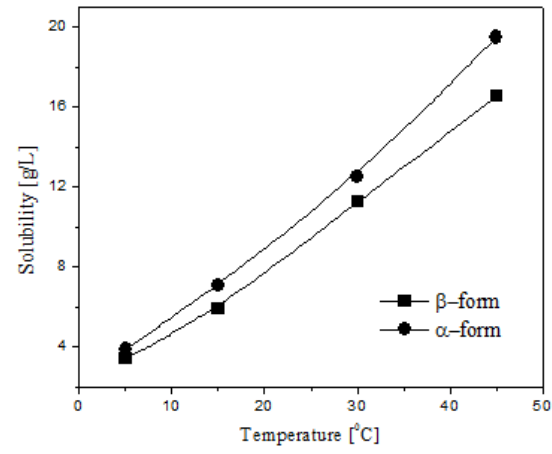

Figure 2. Solubility of $\alpha$-form and $\beta$-form.

In CT crystallizer, even though the phase transformation profile was similar to that of ST crystallizer, as shown in Fig. 4, there was a significant difference between ST and CT crystallizer as regards the time period of phase transformation. That is, the phase transformation completed after $40 \mathrm{~h}$ as using the ST crystallizer, while it required only $20 \mathrm{~h}$ as using the CT crystallizer. This result implied that the Taylor vortices flow of CT crystallizer was more 2.0 times effective than the random fluid motion of ST crystallizer, so the time consumption of crystallization process was remarkably reduced as using the CT crystallizer. The time period of phase transformation of CT and ST crystallizers was also investigated with varying agitation speed, as shown in Fig. 5(a). When varying the agitation speed from $300 \mathrm{rpm}$ to $900 \mathrm{rpm}$, the phase transformation time was decreased from $40 \mathrm{~h}$ to $12 \mathrm{~h}$ as using the ST crystallizer. Yet, it was much more reduced as only changed from $12 \mathrm{~h}$ to $2.5 \mathrm{~h}$ in CT crystallizer, implying that the Taylor vortices flow of CT crystallizer was more effective than the random fluid motion of ST crystallizer in a wide range of agitation speed.

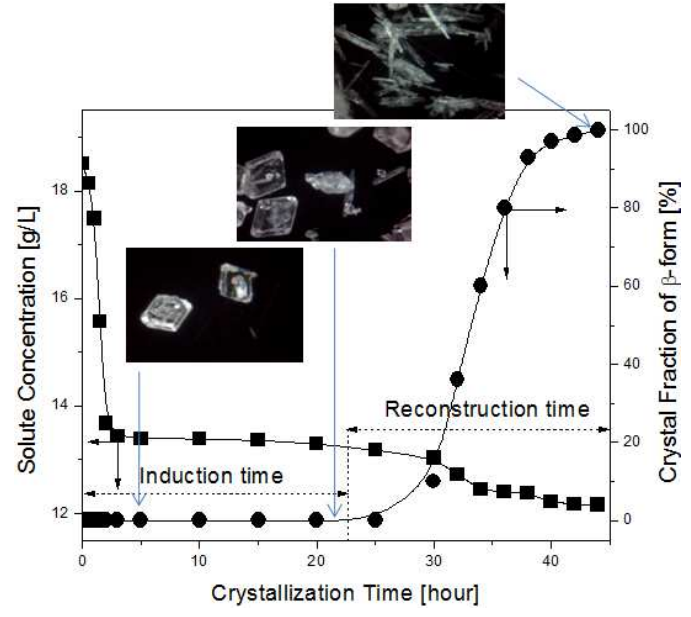

Figure 3. Typical nucleation and reconstruction of $\alpha$-form and $\beta$-form in Stirred tank crystallizer.

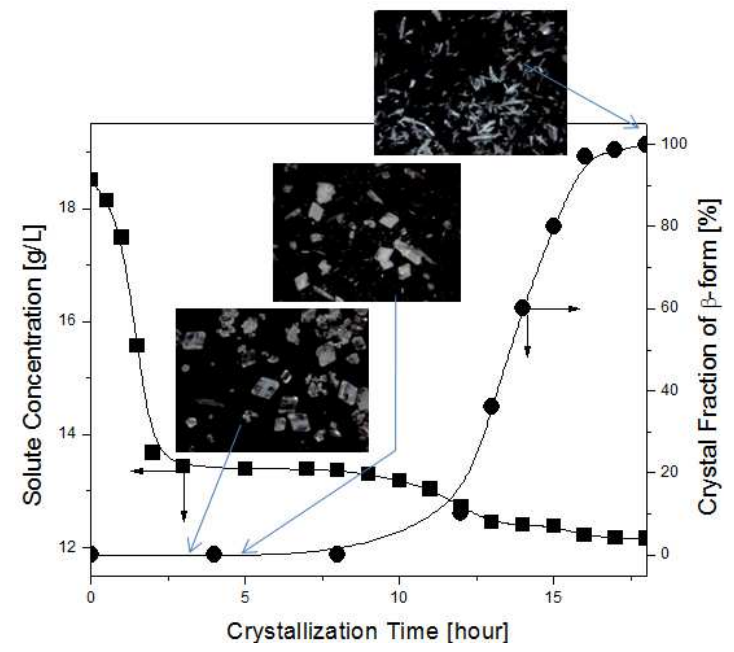

Figure 4. Typical nucleation and reconstruction of $\alpha$-form and $\beta$-form in Couette-Taylor crystallizer. 
(a)
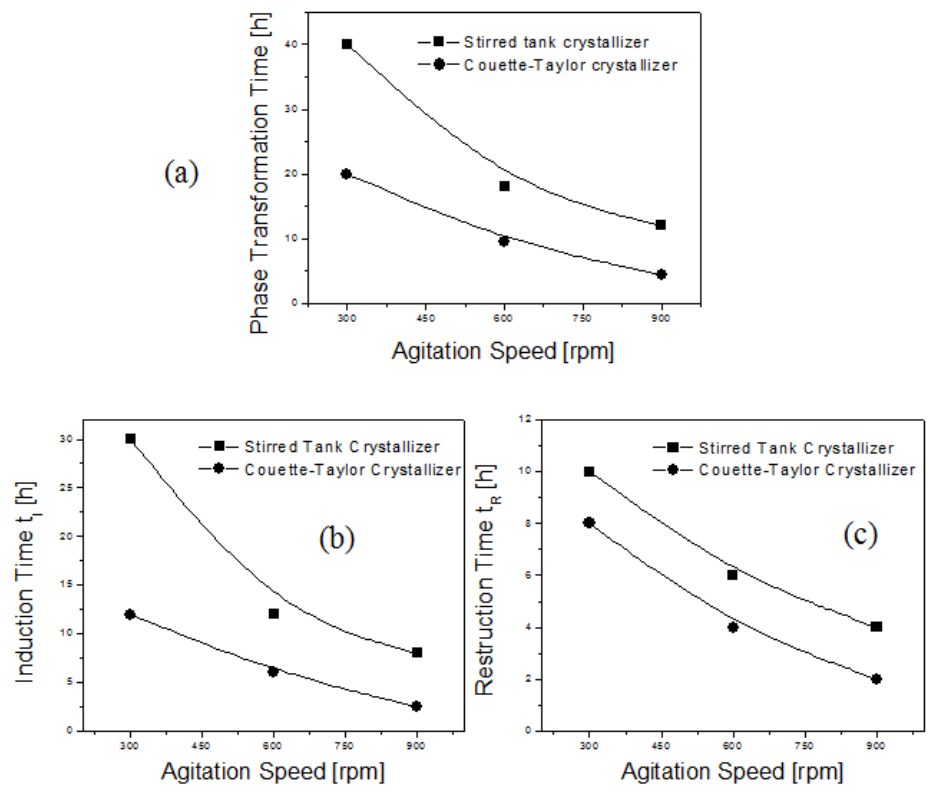

Figure 5. Phase transformation time (a); nucleation time (b) and reconstruction time (c) of L-glutamic acid with varied agitation speed.

In order to understand the mechanism of phase transformation, the phase transformation time was divided into the induction time $\left(\mathrm{t}_{\mathrm{I}}\right)$ and reconstruction time $\left(\mathrm{t}_{\mathrm{R}}\right)$, as described in Fig. 3. Here, the induction time $\left(\mathrm{t}_{\mathrm{I}}\right)$ was defined as the time period of the first nucleation of $\beta$-form, while the reconstruction time $\left(t_{R}\right)$ was the time period from the first nucleation of $\beta$-form to the $100 \%$ wt $\beta$-form of solid product. As such, the induction time $\left(t_{\mathrm{I}}\right)$ corresponded to the nucleation rate of $\beta$-form, while the reconstruction time $\left(t_{R}\right)$ referred to the mass transfer of the dissolution of $\alpha$-form and growth of $\beta$-form.

As shown in Fig. 5(b)-(c), the induction and reconstruction time were illustrated in both CT and ST crystallizer with varied agitation speed. In the ST crystallizer, the induction time $\left(\mathrm{t}_{\mathrm{I}}\right)$ gradually decreased from $30 \mathrm{~h}$ to $8 \mathrm{~h}$ as increasing the agitation speed from $300 \mathrm{rpm}$ to $900 \mathrm{rpm}$, implying that the higher intensity fluid hydrodynamic provided a higher nucleation rate of $\beta$ form, which resulted in a shorter induction time. Yet, when using the CT crystallizer, the induction time $\left(\mathrm{t}_{\mathrm{I}}\right)$ was significantly reduced in a wide range of agitation speed compared to that of the ST crystallizer (Fig. 5(b)). For example, the induction time was $30 \mathrm{~h}$ in ST crystallizer, but it was only $12 \mathrm{~h}$ as using the CT crystallizer at $300 \mathrm{rpm}$ of agitation speed. This result indicated that the nucleation rate of $\beta$-form was more facilitated at least 2.0 times as using the CT crystallizer compared to that of the ST crystallizer. Besides, the reconstruction time $\left(t_{R}\right)$ of phase transformation was also demonstrated in both crystallizers, as depicted in Fig. 5(c). Here, it was also noted that the reconstruction time $\left(\mathrm{t}_{\mathrm{R}}\right)$ was much reduced as using the CT crystallizer, where it only varied from $8 \mathrm{~h}$ to $2 \mathrm{~h}$ as changing the agitation speed from $300 \mathrm{rpm}$ to $900 \mathrm{rpm}$, while it was varied from $10 \mathrm{~h}$ to $4 \mathrm{~h}$ in ST crystallizer. This result indicated that the dissolution rate of $\alpha$ form and growth rate of $\beta$-form were also promoted as using the Taylor vortices flow.

\subsection{Effect of fluid hydrodynamic on nucleation and reconstruction rate}


The influence of fluid hydrodynamic in both crystallizers on nucleation, dissolution and growth of crystal was fundamentally investigated. Here, the nucleation of $\beta$-form can be expressed as using the classical model [1]:

$$
J \propto A \exp \left[-\frac{16 \pi \gamma^{3} v^{2}}{3 k^{3} T^{3}(\ln S)^{2}}\right]
$$

where the induction time $\left(t_{I}\right)$ is inversely proportional to the nucleation rate $(J)$ as $t_{I}=1 / J$. Thus, the induction time $\left(\mathrm{t}_{\mathrm{I}}\right)$ can be correlated with the energy dissipation [6-7]:

$$
\ln t_{I} \propto C-C_{0} \varepsilon
$$

Besides, the dissolution rate of $\alpha$-form and growth rate of $\beta$-form can be expressed as [6]:

$$
\frac{d M_{i}}{d t} \propto S_{A i} k_{i} \Delta C_{i}
$$

From eq (3), the reconstruction time $\left(\mathrm{t}_{\mathrm{R}}\right)$ can be correlated with the energy dissipation as [6 - 7]:

$$
\ln t_{R} \propto-0.21 \ln (\varepsilon)
$$

From the Eq. (2) and (4), the induction and reconstruction time obviously decreased as increasing the energy dissipation. According to Tuan et al [6 - 8], the energy dissipation of Taylor vortices flow in CT crystallizer was always at least 5.0 times higher than that of the random fluid motion in ST crystallizer, so the induction and reconstruction time of phase transformation L-glutamic acid were certainly reduced as using the CT crystallizer.

\section{CONCLUSIONS}

The present study exploited the effective Couette-Taylor crystallization process to promote the nucleation and reconstruction of L-glutamic acid in order to obtain the pure $\beta$-form crystal product in a short crystallization time. Here, the nucleation rate of $\beta$-form and reconstruction rate of $\alpha$-form to $\beta$-form were significantly accelerated at least 2.0 times as using the CT crystallizer compared to that of the ST crystallizer, so the crystallization time remarkably reduced more than 2.0 times as using the CT crystallization process. The advantage of CT crystallizer over the ST crystallizer was explained in term of the higher energy dissipation of Taylor vortices flow in CT crystallizer compared to that of the random fluid motion in ST crystallizer.

Acknowledgment. This research was supported by the Vietnam Academy of Science and Technology (VAST).

\section{REFERENCES}

1. Mullin J. W. - Crystallization. Butterworth-Heinemann, Oxford (1993).

2. Brittain H. G. - Polymorphism in Pharmaceutical Solids. Marcel Dekker Inc, New York, 1999.

3. Florence A. J., Briggs N. E. B., Schacht U., Raval V., McGlone T., and Sefcik J. - The seeded crystallization of $\beta$ L-glutamic acid in a continuous oscillatory baffled crystallizer, J. Org. Process. Res. Dev. 19 (2015) 1903-1911. 
4. Zhang R., Ma C.Y., Liu J. J., and Wang X. Z. - On-line measurement of the real size and shape of crystals in stirred tank crystalliser using non-invasive stereo vision imaging, Chem. Eng. Sci. 137 (2015) 9-21.

5. Ochsenbein D. R., Schorsch S., Salvatori F., Vetter T., Morari M., and Mazzotti M. Modeling the facet growth rate dispersion of $\beta 1$-glutamic acid - Combining single crystal experiments with nD particle size distribution data, Chem. Eng. Sci. 133 (2015) 30-43.

6. Tuan N. A., Joo Y. L. and Kim W. S. - Multiple Feeding Strategy for Phase Transformation of GMP in Continuous Couette-Taylor Crystallizer, Cryst. Growth. Des. 12 (2012) 2780-2788.

7. Tuan N. A., Kim J. M., Chang S. M. and Kim W. S. - Taylor vortex effect on phase transformation of guanosine 5-monophosphate in drowning-out crystallization, Ind. Eng. Chem. Res. 49 (2010) 4865-4872.

8. Tuan N. A., Jeongki K. and Kim W. S. - Noncommon Ion Effect on Phase Transformation of Guanosine 5-Monophosphate Disodium in Antisolvent Crystallization, Ind. Eng. Chem. Res. 54 (2015) 5784-5792.

9. Park S. A., Lee S., and Kim W. S. - Polymorphic Crystallization of Sulfamerazine in Taylor Vortex Flow: Polymorphic Nucleation and Phase Transformation, Cryst. Growth. Des. 15 (2015) 3617-3627.

10. Mayra Q. P. and Kim W. S. - Agglomeration of Ni-Rich Hydroxide in Reaction Crystallization: Effect of Taylor Vortex Dimension and Intensity, Cryst. Growth. Des. 15 (2015) 1726-1734.

11. Phuong N. T. K., Quang K. C., Tuyet N. Đ., Thuy T. T. D., Huyen T. T. T., Huong L. T. T., Dung T. V., Nhan L. T. H., Dung H. T. K., Thao P. T., and Tuan A. T. Polymorphism of L-glutamic Acid: Influence of the Additive Ammonium Sulphate upon the Phase Change between $\alpha$ - and $\beta$-polymorphs, Malay. J. Chem. 17 (2015) 38-46. 